\title{
IS CYSTATIN C TRULY THE BETTER MARKER OF KIDNEY FUNCTION THAN SERUM CREATININ IN SEPTIC NEWBORNS?
}

\author{
I. Maruniak-Chudek, T. Owsianka-Podlesny, J. Wroblewska, D. Jadamus-Niebroj \\ Neonatal Intensive Care, Medical University of Silesia, Katowice, Poland
}

Background and aim: The role of cystatin $\mathrm{C}$ in the diagnosis of reduced kidney function was not unquestionably confirmed in neonates. It was shown that cystatin doesn't cross the placenta and should reflect infant renal function; it's markedly higher concentration was revealed shortly after delivery.

The aim of the study was to analyze the concentrations of cystatin $\mathrm{C}$ in neonates treated for sepsis.

Methods: 32 neonates in the first 14 days of life were enrolled. Serum cystatin concentrations were estimated during the three subsequent days from the admission. 9 neonates developed sepsis, 14 severe sepsis and 9 septic shock.

Results: At admission the mean serum concentration of cystatin was $1.35(1.20-1.49 \mathrm{mg} / \mathrm{dl})$. The lowest concentration $1.13(0.91-1.34 \mathrm{mg} / \mathrm{dl})$ was observed in patients who developed septic shock during the following days. Slightly lower concentrations were found in neonates with sepsis $1.51(1.18-1.84 \mathrm{mg} / \mathrm{dl})$ and severe sepsis $1.38(1.13-1.63 \mathrm{mg} / \mathrm{dl})$. There was no correlation with serum creatinine and gestational age. Significant correlation between the chronological age and cystatin was found $(\mathrm{R}=-0.439, \mathrm{p}=0.01)$. There was a tendency for cystatin decline in patients with sepsis $(1.34$;CI:0.89-1.78) and severe sepsis $(1.24$;CI:1.00$1.48)$, while insignificant increase in patients with septic shock (1.25;CI:0.97-1.53) was revealed. The interrelation between the age and cystatin concentration disappeared in the following days of hospitalization. Even in patients who died in the course of septic shock the observed changes in cystatin were small and not exceeding those of serum creatinine.

Conclusion: Cystatin C shouldn't be estimated in the first days after delivery in neonates. 